\title{
CONTROL DE IRRIGACIÓN DE UN PANEL FOTOVOLTAICO ORIENTADO A LA MAXIMIZACIÓN DEL BENEFICIO ENERGÉTICO
}

\section{IRRIGATION CONTROL OF A PHOTOVOLTAIC PANEL ORIENTED TO THE MAXIMIZATION OF ENERGY BENEFIT}

\author{
Ing. Diego Fernando Rojas Luna, Ing. Miguel Ángel Caro Padilla, \\ MSc. Ricardo Antonio Domínguez Gómez' PhD. Javier Enrique Solano Martínez, PhD. \\ German Alfonso Osma Pinto.
}

* Universidad Industrial de Santander, Bucaramanga, Santander, Colombia. Escuela de Ingeniería Eléctrica, Electrónica y Telecomunicaciones, E-mail: dfr11996@hotmail.com, jesolano@uis.edu.co.

\begin{abstract}
Resumen: Este trabajo busca identificar políticas de control óptimo para maximizar la energía neta generada por un sistema generación compuesto por paneles fotovoltaicos y un sistema de irrigación. Se presenta la formulación del problema de control óptimo y luego se plantea la solución mediante el algoritmo de la programación dinámica. Se muestra la solución para diversos perfiles de irradiancia, velocidad de viento y temperatura ambiente.
\end{abstract}

Palabras clave: Optimización, Irrigación, Paneles Fotovoltaicos, control óptimo.

\begin{abstract}
This paper aims to identify optimal control policies to maximize the net energy generated by s solar generator composed by photovoltaic panels and an irrigation system. The optimal control problem is formulated and its solution is proposed using the dynamic programming algorithm. The solution is presented for three different cases of study with different irradiance, win sped and ambient temperature profiles.
\end{abstract}

Keywords: Optimization, irrigation, photovoltaic panels, optimal control.

\section{INTRODUCCIÓN}

Durante los últimos años en la ciudad, en el país y en el mundo, se han realizado investigaciones con la finalidad de aumentar el rendimiento en los sistemas de generación de energía solar fotovoltaica. Entre las diversas soluciones existentes, varios autores sugieren que la irrigación puede mejorar la producción de energía mediante paneles fotovoltaicos [1-5].

Un sistema de irrigación permite disminuir la temperatura del panel y así aumentar la eficiencia del proceso de conversión de energía. Claramente este sistema de irrigación, ilustrado en la Figura 1 [1], también es un consumidor de energía. Este trabajo busca identificar políticas de control que permitan maximizar la energía neta generada por el sistema de paneles fotovoltaicos. El algoritmo utiliza como insumos tres perfiles: temperatura ambiente, velocidad del viento y la irradiancia. Para solucionar el problema se utiliza el algoritmo de la programación dinámica. Este algoritmo se implementa en el programa MATLAB.

El trabajo se organiza de la siguiente manera: la sección 2 presenta el modelo térmico del panel FV, la sección 3 introduce a la programación dinámica, la sección 4 muestra cómo utilizar la programación dinámica para resolver el problema planteado y la sección 5 explica los análisis de los resultados obtenidos. 


\section{PLANTEAMIENTO DEL PROBLEMA}

Esta sección presenta el planteamiento de los modelos y de la función objetivo. La irrigación se aplica a los paneles FV para disminuir la temperatura, y con ello aumentar la potencia eléctrica generada por el panel FV. La energía neta generada por el sistema descrito se calcula mediante las siguientes ecuaciones:

Donde:

$$
\begin{aligned}
& E_{\text {ben }}=\int_{t \text {-inicial }}^{t-f i n a l} \text { Pbeneficio. } d t \\
& P_{\text {beneficio }}=P_{\text {gen }}-P_{\text {irrigación }}
\end{aligned}
$$

- $t$-inicial : tiempo inicial.

- $t$-final : tiempo final.

- $E_{b e n}$ : Energía neta en un periodo de tiempo.

- $P_{\text {beneficio }}$ : Potencia beneficio.

- $P_{g e n}$ : Potencia generada por el panel FV

- $P_{\text {irrigación }}$ : Potencia consumida por el sistema de irrigación.

La Figura 2 ilustra las variables de entrada, los parámetros y las variables de salida del algoritmo de control, siendo la Potencia de irrigación (Pirrigación) la variable de control.

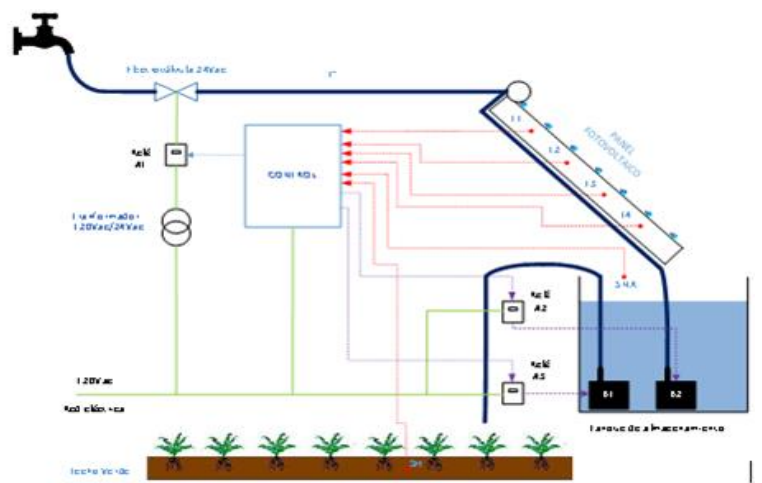

Fig. 1. Sistema de irrigación de un panel FV,

Fig. 2. Algoritmo de control. tomado de [1].

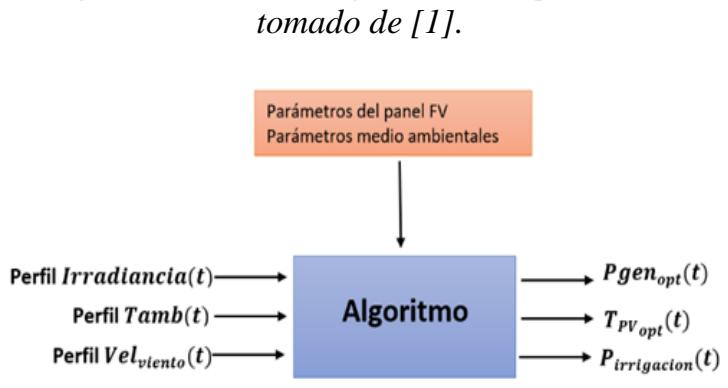

\subsection{Potencia generada}

De acuerdo con [1], la potencia generada es función de la radiación solar incidente y la temperatura de operación del panel FV, por lo tanto, se representa como una fuente de corriente dependiente, existiendo una dependencia mutua entre $T_{p V}$ y $P_{g e n}$, tal como se muestra en la expresión (3).

$$
P_{g e n}=G_{i n E^{*}} A_{p V^{*}} E t a_{0^{*}}\left(1+b_{\cdot}\left(T_{P V}-25\right)\right)
$$

Donde:

- $G_{\text {ine }}$ : Irradiancia, $\left[\frac{W}{m^{2}}\right]$.

- $A_{p V}$ : Área del panel FV, $\left[m^{2}\right]$

- Et $a_{0}:$ Eficiencia del panel FV, [\%].

- $b$ : Coeficiente térmico del panel $\mathrm{FV},\left[\% \frac{\mathrm{K}}{\mathrm{K}}\right]$

\subsection{Modelo térmico del panel fotovoltaico (FV)}

La mayor parte de la radiación solar absorbida por un panel FV no se convierte en energía eléctrica, sino que se convierte en pérdidas que ocasiona un aumento de temperatura del módulo [1]. La curva I-V del módulo, se ve afectada fuertemente por la temperatura, es decir, a medida que la temperatura aumenta el voltaje disminuye, causando una reducción de la potencia generada.

Para este trabajo, se utilizó el modelo presentado por [1]. Este modelo hace una adaptación de un sistema termoeléctrico a un circuito eléctrico y es representado en la Figura 3.

Para este estudio se consideró que el panel FV es un cuerpo muy delgado $(<5 \mathrm{~mm})$ por lo que su temperatura se supone igual en toda su extensión. Por esto el panel es representado por una capacitancia térmica, sin resistencias internas producidas por conducción.

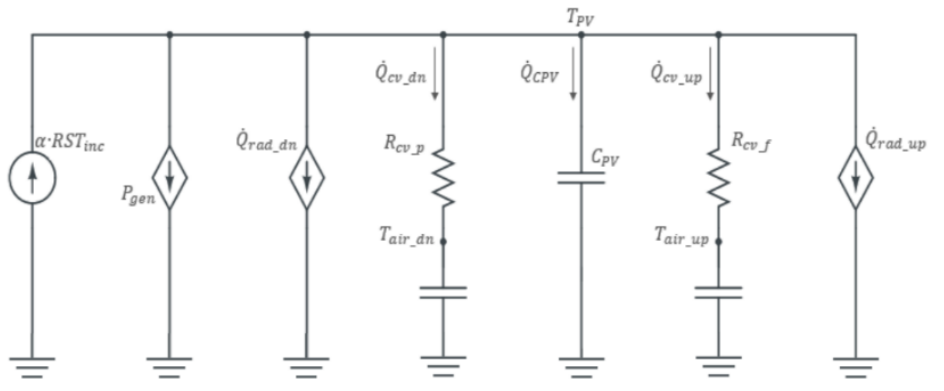

Fig. 3. Modelo térmico RC del panel FV, tomado de [2]. 


\subsubsection{Puntos de temperatura}

El nodo principal es la temperatura de operación del panel FV $\left(T_{P V}\right)$, la cual es una variable de estado. Los nodos de temperatura del aire circundante sobre $\left(T_{\text {air up }}\right)$ y debajo $\left(T_{\text {air } d n}\right)$ de las superficies frontal y posterior del panel FV son representados por capacitancias térmicas, siendo la capacitancia térmica del panel FV. La temperatura del aire por encima y por debajo del panel FV, se consideran sumideros térmicos con capacitancias térmicas de valores muy elevados. Estas dos temperaturas no se consideran iguales, ya que se encuentran a diferentes alturas con respecto al techo. Los objetos que están más cercanos al techo tienden a incrementar su temperatura ya que, el techo tiene una temperatura mayor al ambiente [3].

\subsubsection{Transferencia de calor}

En este modelo se consideró que no existe transferencia de calor por conducción al interior del panel FV, y dado que no hay otro cuerpo a ser analizado en el sistema, no hay transferencia de calor por conducción en este modelo.

Por otro lado, se identifican dos procesos de transferencia de calor por convección entre el panel FV y el medio ambiente para el caso del aire, los cuales ocurren entre las superficies frontal (Rcv $f$ ) y posterior (Rcv p), y el aire circundante a éstas. Se presentan dos temperaturas de aire, ya que éstas pueden depender de la altura de ubicación del panel FV con respecto al techo. Las expresiones (4) y (5) representan la transferencia de calor por convección frontal y posterior, respectivamente.

$$
\begin{aligned}
& Q_{C D f}=h_{C V F} \cdot A_{P V} \cdot\left(T_{P V}-T_{\text {CIY } \text { wD }}\right) \\
& Q_{c V p}=h_{C V p^{*}} A_{P V^{*}}\left(T_{P V}-T_{\text {aIY dn }}\right)
\end{aligned}
$$

Donde:

- $h_{\text {ED } f}$ : Coeficiente de convección posterior, $\left[\frac{W}{m^{2}} \cdot{ }^{D} \mathrm{C}\right]$.

- $\quad h_{\text {ev p }}$ : Coeficiente de convección frontal, $\left[\frac{W}{\mathrm{~m}^{2}} \times{ }^{\circ} \mathrm{C}\right]$.

La transferencia de calor por radiación se representa a partir de una fuente de corriente (flujo de calor) dependiente. En total, se consideran dos fuentes dependientes, una para cada proceso de transferencia ocurrido en las dos superficies del panel FV, superficie frontal y superficie posterior. Cada una de estas tasas de pérdida está conformada por dos componentes debido a la inclinación del panel FV, tal como lo muestran Las expresiones (6) y (7).

$$
\begin{aligned}
& Q_{\text {rad } f}=\varepsilon_{p V f} \cdot \sigma \cdot A_{P V} \cdot\left(F _ { f 1 } \cdot \left(T_{P V}^{4}-\right.\right. \\
& \left.\left.T_{a m b}^{4}\right)+F_{f 2} \cdot\left(T_{p V}^{4}-T_{s k y \text { mod }}^{4}\right)\right) \\
& Q_{\text {rad } p}=\varepsilon_{P V p} \cdot \sigma \cdot A_{P V} \cdot\left(F _ { P 1 } \cdot \left(T_{P V}^{4}-\right.\right. \\
& \left.\left.T_{s k y \text { mod }}^{4}\right)+F_{p 2} \cdot\left(T_{P V}^{4}-T_{a m b}^{4}\right)\right)
\end{aligned}
$$

Donde:

- $\varepsilon_{p V f}$ : Emitancia frontal del panel FV, adimensional.

- $\varepsilon_{P V p}:$ Emitancia posterior del panel FV, adimensional.

- $\sigma$ : Constante de Stefan Boltzmann, $\left[5,67.10^{-8} \frac{W}{m^{2}} \cdot K^{4}\right]$.

- $T_{\text {amb }}$ : Temperatura ambiente, $\left[{ }^{\circ} \mathrm{C}\right]$.

- $T_{\text {skny mod }}$ : Temperatura del cielo ajustada, este ajuste representa el nivel de nubosidad del cielo.

Los factores de visibilidad $f_{f_{1}}, f_{f_{2}}, f_{D_{1}} \mathrm{y} f_{D_{2}}$ de cada superficie del panel FV para el cielo y el techo, se muestran en las siguientes ecuaciones:

$$
\begin{aligned}
& T_{\text {sky mod }}=(1- \\
& \gamma) \cdot 0.052 \cdot T_{a m b}^{\frac{3}{2}}+\gamma \cdot T_{a m b}
\end{aligned}
$$

(8)

$$
\begin{gathered}
f_{f_{1}}=\frac{1}{2} \cdot(1+\cos (180-\delta)) \\
f_{f_{2}}=\frac{1}{2} \cdot(1-\cos (180-\delta)) \\
f_{p 1}=\frac{1}{2} \cdot(1+\cos (180)) \\
f_{p 2}=\frac{1}{2} \cdot(1-\cos (180))
\end{gathered}
$$

Donde:

- $\quad y$ : Coeficiente del nivel de nubosidad.

- $\delta$ : Ángulo de inclinación del panel FV.

\subsubsection{Temperatura del panel FV sin incluir el} sistema de irrigación

En este modelo equivalente, la fuente de corriente independiente representa la irradiación efectiva en el panel FV ( $\left.G_{\operatorname{In} \text { eff }}\right)$ como el producto entre la irradiancia del panel FV (RST ine ) y el coeficiente de transmitancia $(\alpha)$, y la irradiancia del panel FV 
que es el producto entre la radiación solar incidente $\left(G_{\text {Ine }}\right)$ y el área del panel FV $\left(A_{P V}\right)$. Lo anterior, se indica mediante las siguientes expresiones:

$$
\begin{aligned}
& G_{\text {In eff }}=\alpha \cdot R S T_{\text {ine }} \\
& R S T_{\text {ine }}=G_{\text {Ine }} \cdot A_{P V}
\end{aligned}
$$

A continuación, se aplica el análisis de los nodos para obtener la variación del calor del panel FV como se ve en la siguiente expresión (15).

$$
\begin{aligned}
& Q_{\text {CpD }}=G_{\text {in eff }}-P_{\text {gen }}-Q_{\text {rad } f}-Q_{\text {rad } p}- \\
& Q_{C V p}-Q_{C V f}
\end{aligned}
$$

La expresión número (16) permite determinar la temperatura de operación del panel FV en el instante $\mathrm{i}+1$, en función de la temperatura de operación y los flujos de calor en el instante $i \hat{i}$.

$$
\begin{aligned}
& T_{\text {PVi }+1}=T_{P V}+\frac{\Delta t}{G_{P A} A_{P V}} \cdot\left(G_{\text {in eff i }}-P_{\text {gen }}-\right. \\
& \left.Q_{\text {rad } f i}-Q_{\text {radpi }}-Q_{\text {conv pi }}-Q_{\text {conv } f i}\right)
\end{aligned}
$$

Donde:

- $C_{\mathrm{p}}$ : Capacidad térmica del panel FV, $\left[\frac{\mathbb{J}}{\mathrm{m}^{2}} \times K\right]$.

- $\Delta t$ : Paso de tiempo, segundos.

\subsubsection{Comportamiento térmico del agua de riego}

La película de agua durante el proceso de irrigación obstruye la interacción de la superficie superior del panel con el entorno, dado que elimina la transferencia de calor por radiación y convección frontal. En consecuencia, se produce una corriente o flujo de calor de enfriamiento hacia el panel FV como se indica en la expresión (17).

$$
Q_{\text {enfriamiento }}=\frac{T_{P V}-T_{\text {agua } r}}{R r g}
$$

Donde:

- $T_{\text {aguar }}$ : Temperatura del agua de riego, $\left[{ }^{\circ} \mathrm{C}\right]$.

- $\operatorname{Rrg}:$ Resistencia de riego, $\left[\frac{W}{{ }_{G}}\right]$.

\subsubsection{Temperatura del panel FV incluyendo el sistema de irrigación}

La variación del calor del panel FV se ve afectada. Por consiguiente, con el análisis de nodos se obtiene la expresión (18). Y la temperatura de operación se representa en la expresión (19).

$$
\begin{aligned}
& Q_{\text {cpD }}=G_{\text {In eff }}-P_{\text {gen }}-Q_{\text {radf }}-Q_{\text {rad p }}- \\
& Q_{\odot v p}-Q_{\varpi V f}-Q_{\text {enfriamiento }} \\
& T_{\text {PVi }+1}=T_{P V i}+\frac{\Delta t}{G_{P A P V}} \cdot\left(G_{\text {In eff i }}-P_{\text {gen i }}-\right. \\
& Q_{\text {rad fi }}-Q_{\text {radpi }}-Q_{\text {conv pi }}-Q_{\text {convf i }}- \\
& \text { Qenfriamiento i) }
\end{aligned}
$$

Aunque hay expresiones que determinan el comportamiento de la temperatura del agua de riego y la temperatura del agua en la cava, como se muestra en [2] y [5], en el caso de estudio, se asume que estas temperaturas son iguales y tienen un comportamiento igual a la temperatura ambiente.

\section{PROGRAMACIÓN DINÁMICA}

Richard Bellman [10] en los años cincuenta desarrolló la programación dinámica y, en esta postuló el principio de optimalidad que dice: "Una política óptima tiene la propiedad de que cualesquiera que sean su estado y decisión iniciales, las decisiones subsecuentes deben constituir una política óptima con respecto al estado resultante de la decisión inicial.”. La programación dinámica es un proceso de decisión de optimización por etapas, que permite solucionar problemas en los cuales es necesario tomar decisiones en etapas consecutivas, este se basa en el método propuesto por Bellman en [11].

\subsection{Algoritmo de programación dinámica}

Sea $\pi=\left\{\mu_{0}, \mu_{1, m \ldots o} \mu_{N-1}\right\}$ una política de control [11], usando el estado inicial $T_{P V O}$ se tiene:

$l_{\mathbb{M}}\left(x_{\mathbb{Q}}\right)=g_{\mathbb{N}}\left(x_{\mathbb{N}}\right)+\sum_{k=0}^{N-1} h_{k}\left(T_{k^{s}}, \mu_{k}\left(T_{K}\right)\right)$

Donde:

- $\vartheta_{\mathrm{N}}\left(T_{\mathrm{N}}\right)$ : Costo final.

- $h_{k}\left(T_{k}, \mu_{k}\left(T_{P V K}\right)\right)$ : El costo de aplicar el control.

La función se basa en el principio de optimalidad [10], el algoritmo de programación dinámica DP evalúa el costo óptimo de la función $l_{k}\left(T_{P V}{ }^{\mathbb{T}}\right)$ en cada nodo que se encuentra discretizado tiempoespacio del estado, se procede hacia atrás en el tiempo [7]. 
Cálculo del costo final

$$
l_{N}\left(T^{\mathrm{i}}\right)=g_{N}\left(T_{P V}{ }^{i}\right)
$$

Cálculo intermedio $k=N-1 \propto 0$

$$
l_{k}\left(T^{i}\right)=\max _{\mathrm{uk}}\left\{h_{k}\left(T_{P V}, u_{k}\right)+\left(F_{k}\left(T_{P V}, u^{i}, u_{k}\right)\right)\right\}
$$

Donde:

- $T_{P V}{ }^{\mathbb{i}}$ : Índice de tiempo.

- $k$ : Discretización tiempo-estado del espacio.

- $T_{k+1}$ : Función de costo para llegar al siguiente nodo.

La salida de la función $\left(F_{k}\left(T_{P V}{ }^{\frac{i}{a}}, u_{k}\right)\right)$ es una variable continua en el espacio de estado que está entre punto y punto.

La salida del algoritmo (20) - (21) es el control óptimo valido para cualquier estado y tiempo en el intervalo estudiado.

\subsection{Formulación del problema}

Investigadores de la Universidad ETH de Zúrich [6] comparten una función que permite solucionar problemas de optimización utilizando el método de la programación dinámica. Este tipo de problemas se pueden resolver de la siguiente manera.

La variable de estado es la temperatura del panel FV. La variable de control es la potencia consumida por el sistema de irrigación. El problema de control óptimo en tiempo discreto se basa en maximizar la potencia beneficio durante un periodo de tiempo, por lo cual se puede describir como:

$$
\begin{aligned}
& \max _{\mathrm{Uk}} \sum_{\mathrm{K}=1}^{W-1} P_{\text {gen }}-P_{\text {Irrigacion }} \\
& \mathrm{T}_{\mathrm{PVk}+1}=\mathrm{f}\left(\mathrm{T}_{\mathrm{PVk}}, \mathrm{u}_{\mathrm{k}}\right)+\mathrm{T}_{\mathrm{PVk}} \\
& \mathrm{T}_{\mathrm{PV}}\left(\mathrm{t}_{\text {inicial }}\right)=\mathrm{T}_{\mathrm{PWo}} \\
& \text { T_PV } \in \text { [T_min, T_max] } \\
& \mathrm{N}=12 \text { horas } * \frac{60 \text { minutos }}{1 \text { hora }}+1 \\
& l(u(t))_{k}= \\
& \int_{t_{\text {inivinal }}^{t_{\text {final }}}}^{\left(P_{g e n}\right.}\left(I(t), T_{P V}\left(T_{\text {amb }}(t), u(t)\right)\right)- \\
& \left.P_{\text {irri }}(u(t))\right) d t
\end{aligned}
$$

Donde:

- $T_{P V}\left(T_{a m b}(t), u(t)\right)$ : Temperatura del panel FV

- $T_{a m b}(t)$ : Temperatura ambiente.

\section{CASO DE ESTUDIO}

Para el caso de estudio se utilizaron valores de irradiancia, temperatura ambiente y velocidad del viento, medidos en la escuela de ingeniería

\begin{tabular}{|c|c|}
\hline Parámetro & Interpretación \\
\hline Pnom: $250[\mathrm{~W}]$ & Potencia nominal del panel \\
\hline$A_{P V}: 1.6\left[m^{2}\right]$ & Área del panel \\
\hline Eta $_{0}: \frac{p_{\text {nnom }}}{A p^{*} 1000}[\%]$ & Eficiencia del panel FV \\
\hline$b:-0.0043\left[\frac{96}{K}\right]$ & Coeficiente térmico del panel FV \\
\hline$\alpha: 0.9$ & Coeficiente de transmitancia \\
\hline$C_{p}: 10140\left[\frac{J}{m^{2}}, K\right]$ & Capacidad térmica del panel FV \\
\hline $\begin{array}{c}\sigma: \\
5.67 .10^{-8}\left[W / m^{2} . K^{4}\right. \\
] .\end{array}$ & Constante de Stefan Boltzmann \\
\hline$\delta: 10 \frac{p I}{180}$ & $\begin{array}{l}\text { Ángulo de inclinación del panel } \\
\text { FV }\end{array}$ \\
\hline$G_{e x t}: 1278\left[\frac{W}{m^{2}}\right]$ & Irradiancia máxima \\
\hline $\operatorname{Rrg}: 0.00285\left[\frac{\mathrm{W}}{9 \mathrm{C}}\right]$ & Resistencia de riego \\
\hline$\varepsilon_{p D_{f n g}}: 0.9$ adimensional & Emitancia del agua \\
\hline $\operatorname{Rrg}: 0.00285 / 5\left[\frac{W}{96}\right]$ & Resistencia de mezclado \\
\hline$V_{r g}: 4,5[\mathrm{~L} / \mathrm{m}]$ & Volumen de agua de riego \\
\hline$C_{\text {agua: }}: 4180\left[\frac{f}{K}\right]$ & Capacidad específica del agua \\
\hline$k m_{e}=3.5\left[\frac{{ }^{2} \bar{E}}{W}\right]$ & $\begin{array}{c}\text { Coeficiente de enfriamiento } \\
\text { dentro de la cava }\end{array}$ \\
\hline $\mathrm{km}_{\mathrm{r}}: 1 / \mathrm{km}_{\mathrm{c}}\left[\frac{W}{\mathrm{q}_{\mathrm{C}}}\right]$ & Resistencia de enfriamiento \\
\hline
\end{tabular}
eléctrica, electrónica y telecomunicaciones de la Universidad Industrial de Santander, campus principal en la ciudad de Bucaramanga.

\subsection{Parámetros}

Los parámetros corresponden a los paneles FV ubicados en la terraza del edificio de ingeniería eléctrica, electrónica y telecomunicaciones de se presentan en la Tabla 1 [1].

Tabla 1: Parámetros de simulación

La discretización del problema se realiza mediante tres vectores os vectores $v x$ y $v u$ :

$v T_{P V}$ : Es el vector de estado, cuenta con delta de temperatura de $0.1{ }^{\circ} \mathrm{C}$, su límite inferior de $20^{\circ} \mathrm{C}$ y su límite superior es $80{ }^{\circ} \mathrm{C}$. vu: Es el vector de control, es la potencia consumida por el sistema de irrigación. Donde, la potencia mínima a $0 \mathrm{~W}$ corresponde a la potencia cuando está desactivado el sistema, y la potencia máxima a $15 \mathrm{~W}$ es la potencia cuando el sistema está activado. Finalmente, $v T$ : Es la variable independiente. Donde, 0 corresponde a las 6 am con un delta de 
tiempo de 60 segundos, hasta 43200 segundos que pertenece a las $6 \mathrm{pm}$.

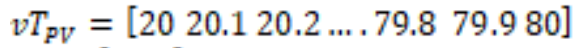

$$
\begin{aligned}
& \mathrm{vu}=\left[\begin{array}{ll}
0 & 15
\end{array}\right]
\end{aligned}
$$

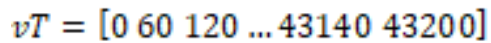

\subsection{Resultados}

Para ilustrar la utilidad del algoritmo, se muestran resultados obtenidos considerando como insumo las variables medidas el día 22 de marzo de 2018. Estas condiciones climáticas, temperatura ambiente (Tamb), irradiancia (Ginc) y velocidad del viento (Vel) se ilustran en la figura 4. El día seleccionado corresponde al primer trimestre del 2018.

Las variables analizadas fueron: Potencia consumida por el sistema de irrigación, temperatura del panel $\mathrm{FV}$ con irrigación y sin irrigación, potencia generada por el panel FV con irrigación, sin irrigación y potencia beneficio.

En la Figura 5 se observa la potencia consumida por el sistema de irrigación. En la Figura 6 se presentan las variaciones de la temperatura que presenta el panel FV. La Figura 7 muestra la potencia beneficio con respecto a la potencia sin irrigación. Posteriormente, en la Figura 8 se observa un zoom respecto a la potencia de beneficio. Finalmente, en la Figura 9, se presenta la variación de energía beneficio que presenta un incremento porcentual de $3.36 \%$ ante la energía generada sin irrigación.

En la Figura 5, se ilustra la potencia consumida por el sistema, y los momentos del día que el sistema entró en operación los cuales se concentran principalmente entre las 8 am a las 10:30 am y entre las 11:30 am a 12:30 pm.

La Figura 6 presenta tres temperaturas: La temperatura ambiente es la de valores más bajos, la temperatura de irrigación con valores cercanos a la temperatura ambiente, y la temperatura $\sin$ irrigación es la que alcanza valores cercanos a los $60^{\circ} \mathrm{C}$. La Figura 7, se ilustra la comparación de las potencias generadas con y sin el sistema de irrigación.

En la Figura 8, se observa de forma más detallada la potencia beneficio generada sin la irrigación. En la Figura 9, se observa la energía beneficio vs. la energía sin irrigación, para este día de operación, se calculó la variación porcentual de energía para este día y presentó un incremento de $3.36 \%$, teniendo así un mayor incremento en la energía beneficio del panel FV.

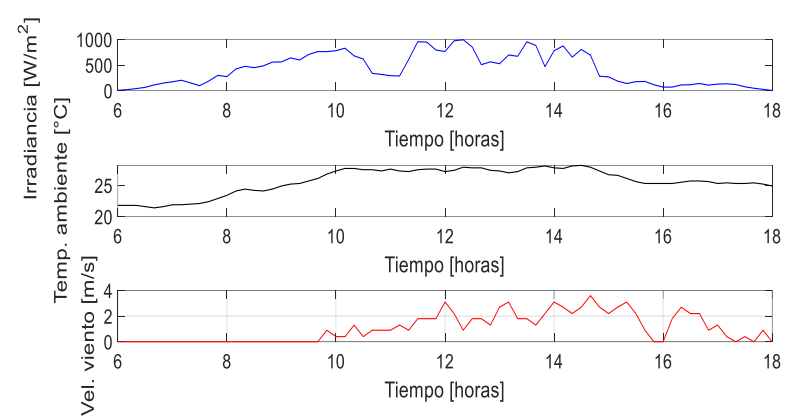

Fig. 4. Perfil de irradiación, Temperatura ambiente, Velocidad del viento.

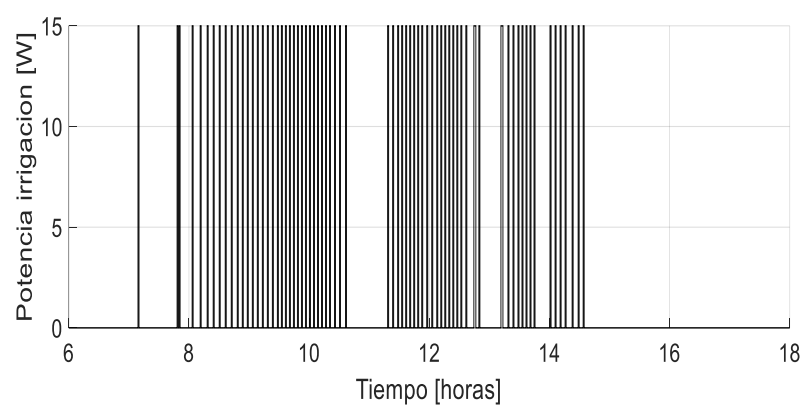

Fig. 5. Potencia irrigación

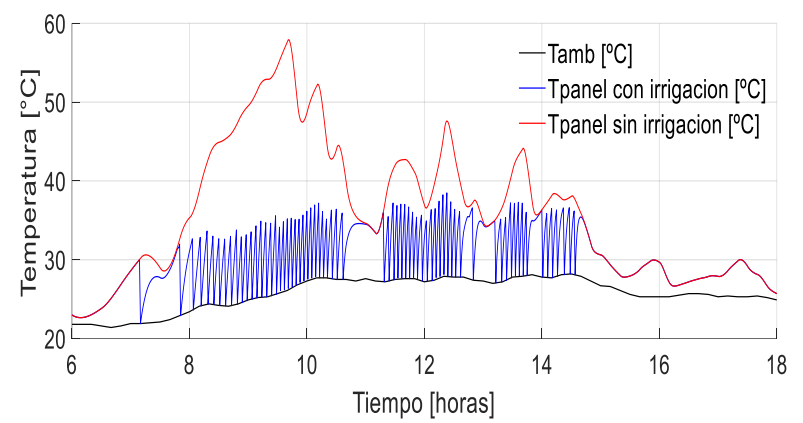

Fig. 6. Perfil de temperatura ambiente, del panel con y sin irrigación.

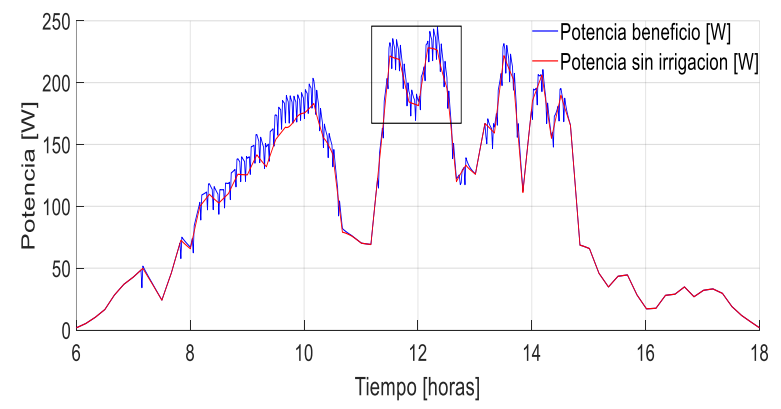

Fig. 7. Potencia beneficio vs sin irrigación. 


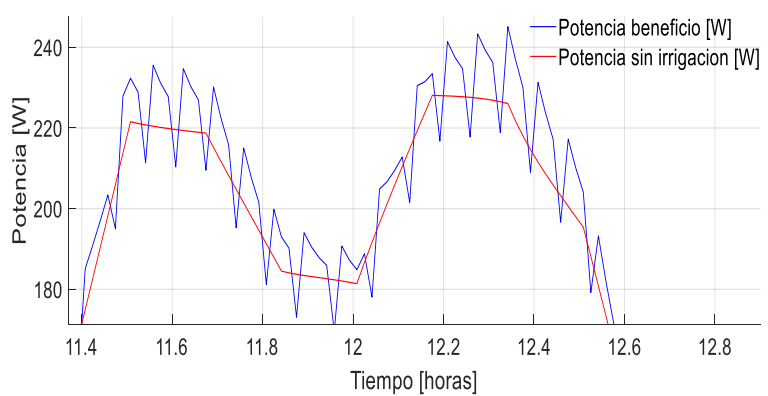

Fig. 8. Zoom potencia beneficio vs sin irrigación.

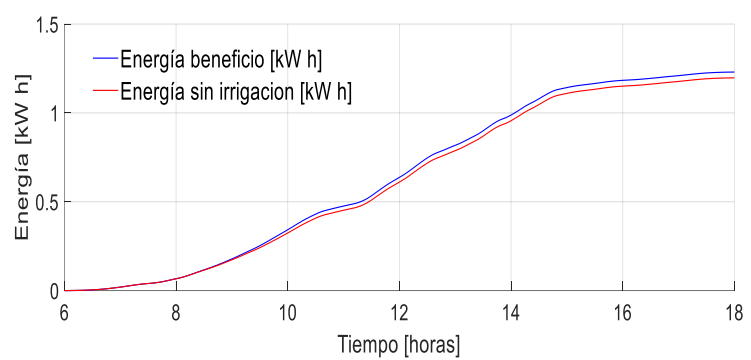

Fig. 9. Energía beneficio vs sin irrigación.

Se aplica el mismo algoritmo a días con condiciones ambientales diferentes en un clima tropical para el primer trimestre del 2018. En cada caso de estudio se obtuvo un incremento de la energía neta que oscila entre $2.13 \%$ a $3.36 \%$ empleando el sistema de irrigación, en comparación al sistema sin irrigación.

\section{CONCLUSIONES}

Se diseñó un algoritmo basado en la programación dinámica haciendo uso del programa MATLAB, el cual permitió realizar la optimización del tiempo de irrigación de los paneles fotovoltaicos a partir de un modelo térmico RC.

Según los resultados obtenidos se concluye que la simulación realizada mediante el método de programación dinámica funciona eficazmente para las diferentes condiciones ambientales que se presentan en un clima tropical para el primer trimestre del 2018, en cada caso de estudio se obtuvo un incremento de la energía neta de $3.36 \%$ empleando el sistema de irrigación, en comparación al sistema sin irrigación.

Se comprobó que los resultados obtenidos a partir de la simulación son los esperados, puesto que la activación de este sistema de irrigación permitió una mayor generación de energía eléctrica, mejorando la cantidad de energía entregada por un panel FV.

\section{REFERENCIAS}

Osma, G. (2015). "Caracterización del desempeño de paneles FV en terrazas de edificaciones localizadas en entornos con clima tropical cálido". Tesis doctoral: Universidad Industrial De Santander. pp. 4-13, 13- 15.

Ortiz, A, Jimenez, G. (2013). "Modelamiento térmico de un panel fotovoltaico con disipador de calor operando en el norte de chile". Universidad De Chile. pp. 12-20.

Rojas, W y Flórez, C. (2016). “Impacto de dos estrategias de irrigación en la potencia generada y la temperatura de operación de un panel fotovoltaico upsolar $250 \mathrm{~W}$ instalado sobre techo verde en la ciudad de Bucaramanga" Universidad Industrial de Santander, pp. 23-27.

Jaimes, J y Cortés, C. (2018). "Estudio del balance térmico de los paneles fotovoltaicos instalados en la terraza del edificio de ingeniería eléctrica". Universidad Industrial De Santander. pp. 12-21, 89-95.

Hernández, E, Quintero, L y Torres, M. (2014). "Caracterizacion de la operación de paneles fotovoltaicos en condiciones tropicales bajo la influencia de un techo verde. Caso Bucaramanga". Universidad Industrial De Santander. pp. 42-45.

Guirguis, A. Ruiz E, Piliougine, M y Sidrach-deCardona, M. (2007). Estudio de la temperatura de módulos fotovoltaicos bajo diferentes condiciones ambientales: Modelos NOTC y balance de energía.

Bellman, R, (1957). Dynamic Programming. Princeton University Press, Princeton. pp. 83. Nueva Jersey.

Sundström, Olle y Guzzella, Lino. (2009). "A Generic Dynamic Programming Matlab Function". IEEE, 1625-1630. 